\title{
Preparation, characterization, and in vivo evaluation of a self-nanoemulsifying drug delivery system (SNEDDS) loaded with morin-phospholipid complex
}

\author{
This article was published in the following Dove Press journal: \\ International Journal of Nanomedicine \\ 17 December 2011 \\ Number of times this article has been viewed
}

\author{
Jinjie Zhang' \\ Qiang Peng' \\ Sanjun Shi' \\ Qiang Zhang ${ }^{2}$ \\ Xun Sun' \\ Tao Gong' \\ Zhirong Zhang' \\ 'Key Laboratory of Drug Targeting \\ and Drug Delivery System, Ministry \\ of Education, West China School \\ of Pharmacy, Sichuan University, \\ Chengdu, People's Republic of China; \\ ${ }^{2}$ State Key Laboratory of Natural \\ and Biomimetic Drugs, School of \\ Pharmaceutical Sciences, Peking \\ University, Peking, People's \\ Republic of China
}

Correspondence: Tao Gong;

Zhirong Zhang

Key Laboratory of Drug Targeting and Drug Delivery System, Ministry of Education, West China School of Pharmacy, Sichuan University, No 17, Section 3, Southern Renmin Road, Chengdu 61004I, People's Republic of China

Tel +862885501615

Fax +86288550 I6I5

Email gongtaoy@126.com;

zrzzl@vip.sina.com
Background: As a poorly water-soluble drug, the oral application of morin is limited by its low oral bioavailability. In this study, a new self-nanoemulsifying drug delivery system (SNEDDS), based on the phospholipid complex technique, was developed to improve the oral bioavailability of morin.

Methods: Morin-phospholipid complex (MPC) was prepared by a solvent evaporation method and characterized by infrared spectroscopy and X-ray diffraction. After formation of MPC, it was found that the liposolubility of morin was significantly increased, as verified through solubility studies. An orthogonal design was employed to screen the blank SNEDDS, using emulsifying rate and particle size as evaluation indices. Ternary phase diagrams were then constructed to investigate the effects of drug loading on the self-emulsifying performance of the optimized blank SNEDDS. Subsequently, in vivo pharmacokinetic parameters of the morin-phospholipid complex self-nanoemulsifying drug delivery system (MPC-SNEDDS) were investigated in Wistar rats (200 mg/kg of morin by oral administration).

Results: The optimum formulation was composed of Labrafil ${ }^{\circledR}$ M 1944 CS, Cremophor ${ }^{\mathbb{R}}$ RH 40, and Transcutol ${ }^{\circledR} \mathrm{P}(3: 5: 3, \mathrm{w} / \mathrm{w})$, which gave a mean particle size of approximately $140 \mathrm{~nm}$. Oral delivery of the MPC-SNEDDS exhibited a significantly greater $\mathrm{C}_{\max }(28.60 \mu \mathrm{g} / \mathrm{mL})$ than the morin suspension $(5.53 \mu \mathrm{g} / \mathrm{mL})$ or MPC suspension $(23.74 \mu \mathrm{g} / \mathrm{mL})($ all $P<0.05)$. $\mathrm{T}_{\text {max }}$ was prolonged from 0.48 to 0.77 hours and to 1 hour for MPC and MPC-SNEDDS, respectively. In addition, the relative oral bioavailability of morin formulated in the MPC-SNEDDS was 6.23-fold higher than that of the morin suspension, and was significantly higher than that of the MPC suspension $(P<0.05)$.

Conclusion: The study demonstrated that a SNEDDS combined with the phospholipid complex technique was a promising strategy to enhance the oral bioavailability of morin.

Keywords: morin, phospholipid complex, self-nanoemulsifying drug delivery system, oral bioavailability

\section{Introduction}

Morin $\left(3,5,7,2^{\prime}, 4^{\prime}\right.$-pentahydroxyflavone) is one of the flavonols that has been identified in fruits, vegetables, tea, and many medicinal herbs from Asia. ${ }^{1}$ It has been reported to possess anti-inflammatory, anticancer, antioxidant, antihypertensive, and anticlastogenic activities. ${ }^{2-6}$ It has also been found to have an inhibitory effect on xanthine oxidase. ${ }^{7}$ In addition, in a toxicity study of dietary administered morin in rats, no mortality or abnormal clinical signs were shown. ${ }^{8}$ 
However, available pharmacokinetic data in both humans and rats are scarce at present. A previous study compared the pharmacokinetics between morin and quercetin after oral administration but failed to consider the absolute bioavailability of morin. ${ }^{9}$ As a poorly water-soluble drug, the absolute bioavailability of morin after a single oral dose is very low $(<1 \%)$, as determined in the present study. To the best of the authors' knowledge, low oral bioavailability may result in decreased efficacy when therapeutic plasma levels are not achieved, or may result in unanticipated toxicity at a high dose of morin. However, to date, few studies have focused on the applicable formulation of morin through oral administration. Most studies have used a simple morin aqueous solution via parenteral route to obtain the targeted pharmacological effect. ${ }^{5,6}$ Parenteral administration offers the best absorption but has obvious disadvantages, such as low patient compliance, safety considerations, and high medication costs. ${ }^{10}$ Thus, there is a pressing need to develop a new oral dosage form of morin with improved bioavailability and, furthermore, to obtain successful therapeutic effects at a decreased oral dose.

Various formulation techniques have been developed to improve the bioavailability of poorly water-soluble drugs, such as solid dispersion, cyclodextrins, emulsions, liposomes, and nanoparticles. ${ }^{11}$ Among these methods, much attention has recently been focused on self-nanoemulsifying drug delivery systems (SNEDDSs) to improve the oral bioavailability of poorly soluble drugs. ${ }^{12-16}$ For SNEDDSs, isotropic mixtures of oils and surfactants are used to form fine oil-in-water nanoemulsions when exposed to aqueous media, such as gastrointestinal fluids under mild agitation with droplet size less than $200 \mathrm{~nm} .{ }^{17}$ Drug delivery advantages offered by SNEDDSs include presenting and maintaining the drug in a dissolved rate or in small droplets of oil; protection against enzymatic hydrolysis; the potential for enhanced absorption afforded by surfactant-induced permeability changes; and the inhibitory effect on P-glycoprotein (P-gp) activities. ${ }^{18}$ This renders the SNEDDS as a good candidate for oral delivery of morin in an attempt to solve its bioavailability problem.

Previous studies have demonstrated that phospholipid complexes can improve the liposolubility and the therapeutic efficacy of certain drugs with poor oral bioavailability. ${ }^{19-21}$ In this study, a phospholipid complex was prepared to improve the liposolubility of morin and facilitate the incorporation of morin into a SNEDDS.

Thus, the purpose of this study was to develop a novel SNEDDS based on the phospholipid complex technique to enhance the oral bioavailability of morin. This is the first time, to the authors' knowledge, that an oral formulation of morin was developed to address its bioavailability problem. It is hoped that this study not only offers a good example of enhancing the oral bioavailability of poorly water soluble drugs by the combined use of phospholipid complex and a SNEDDS, but also provides a promising oral formulation of morin for clinical application.

\section{Materials and methods Materials}

Morin hydrate was purchased from Sigma-Aldrich ${ }^{\circledR}\left(\operatorname{Sigma}^{\circledR}\right.$ Chemical, St Louis, MO). Polyoxyl 40 hydrogenated castor oil (Cremophor ${ }^{\circledR}$ RH 40), and polyoxyl 35 castor oil (Cremophor ${ }^{\circledR}$ EL) were donated by BASF (Ludwigshafen, Germany). Oleoyl macrogolglycerides (Labrafil ${ }^{\circledR}$ M 1944 CS), propylene glycol laurate (LauroglycolTM FCC), propylene glycol monolaurate (Lauroglycol ${ }^{\mathrm{TM}}$ 90), and diethylene glycol monoethyl ether (Transcutol ${ }^{\circledR} \mathrm{P}$ ) were kindly supplied by Gattefossé China Trading Company (Shanghai, People's Republic of China). Acetonitrile of high performance liquid chromatography (HPLC) grade was purchased from SK Chemicals (Seoul, South Korea). Soya phospholipid was obtained from Lipoid (Ludwigshafen, Germany). Benzoic acid and 1,2-propylene glycol were purchased from Ruijinte Chemical Co, Ltd (Tianjin, China). Tetrahydrofuran was purchased from Bioway America (Marlton, NJ). Carboxymethyl cellulose sodium, hydrochloric acid, polyoxyethylene sorbitan monooleate $\left(\right.$ Tween $^{\circledR} 80$ ), ethanol, and phosphoric acid were obtained from Kermel Chemical Reagent Co, Ltd (Tianjin, China). All other chemicals were of analytical reagent grade.

\section{Preparation of morin-phospholipid complex}

The morin-phospholipid complex (MPC) was prepared with morin and phospholipid at a mass ratio of $1: 1.5$. Required amounts of morin and phospholipids were placed in a $100-\mathrm{mL}$ round bottom flask, and $30 \mathrm{~mL}$ tetrahydrofuran was added. The mixture was then stirred with a magnetic agitator (Yuhua Instrument Co, Ltd, Gongyi, China) at $40^{\circ} \mathrm{C}$ for 2 hours. Afterwards, a vacuum rotary evaporator (Büchi Rotavapor ${ }^{\circledR}$ R-3; Büchi, Switzerland) was used to evaporate tetrahydrofuran, and the residue was dried under vacuum for 24 hours. The resultant MPC was transferred into a glass bottle and stored at room temperature. 


\section{Characterization of MPC}

MPC was verified by Fourier transform infrared spectrophotometry (FT-IR Spectrometer IFS-55; Bruker, Fällanden, Switzerland) and X-ray diffractometry (D/max-r A; Rigaku Denki, Tokyo, Japan). The infrared spectra of morin, MPC, and physical mixture of morin and phospholipids were obtained by the $\mathrm{KBr}$ method. The $\mathrm{X}$-ray diffractogram was scanned with the diffraction angle increasing from $0^{\circ}$ to $60^{\circ}, 2 \theta$ angle, with a step angle of $0.02^{\circ}$ and a count time of 0.40 seconds.

\section{Determination of morin content in MPC}

The content of morin in the complex was determined by HPLC method, established according to a previous study with some modifications. ${ }^{9}$ The analysis was performed on a Waters ${ }^{\circledR} 2690$ HPLC system equipped with a Waters 996 Photodiode Array Detector (Waters, Milford, MA). A Zorbax SB-C18 (Agilent Technologies, Santa Clara, CA) analytical column $(150 \mathrm{~mm} \times 4.6 \mathrm{~mm}, 5 \mu \mathrm{m})$ was used with a mobile phase consisting of acetonitrile and $0.5 \%$ phosphoric acid $(24: 76, \mathrm{v} / \mathrm{v})$ by a flow rate of $1.0 \mathrm{~mL} / \mathrm{min}$. Detection wavelength was $252 \mathrm{~nm}$, and the injection volume was $100 \mu \mathrm{L}$.

Studies showed that the precision, accuracy, and recovery of this HPLC method all met the measurement requirements. The linear standard curve $(r=0.9999)$ was used to determine the concentration of morin. All measurements were done in triplicate.

\section{Solubility studies}

Solubility studies were carried out to verify whether the liposolubility of morin was improved after formation of MPC. Excess amounts of morin, MPC, and the physical mixture of morin and phospholipid were added to $1 \mathrm{~mL}$ of water or $\mathrm{n}$-octanol in sealed glass containers. The samples were kept at $25^{\circ} \mathrm{C}$ with constant shaking for 48 hours and then centrifuged at $5064 \times \mathrm{g}$ for 10 minutes using a table-top, high-speed centrifuge (TGL-16G; Xingke Scientific Instruments Co, Ltd, Hunan, China). Afterward, supernatants were removed and suitably diluted with methanol. The concentration of morin was determined by the HPLC method mentioned above. Each experiment was run in triplicate. Similarly, the solubilities of morin and MPC in three different oils (Labrafil M 1944 CS, Lauroglycol FCC, and Lauroglycol 90) were determined.

\section{Formulation optimization of a blank SNEDDS}

The blank SNEDDS formulation was screened by the orthogonal design using Labrafil M 1944 CS, Lauroglycol 90, and Lauroglycol FCC as oil phase; Cremophor EL, Cremophor RH 40, and Tween 80 as surfactants; and Transcutol P and 1,2-propylene glycol as cosurfactants. Eighteen different combinations were obtained, and nine experiments were then performed for each combination. Mixtures of oil, surfactants, and cosurfactants at prefixed ratios were placed in tubes and vortexed vigorously. After that, certain amounts of the mixtures were added to distilled water ( $\mathrm{v} / \mathrm{v}, 1: 100)$. The mean particle size of the samples was determined by a photon correlation spectroscope (Malvern Zetasizer Nano ZS90, Malvern Instruments Ltd, Worcestershire, UK). All measurements were done in triplicate. The system composed of Labrafil M 1944 CS, Cremophor RH 40, and Transcutol P was further studied using the particle size and emulsification rate as indices.

\section{Construction of ternary phase diagram}

The self-emulsifying region of the selected system with different drug loading was identified from ternary phase diagrams. A series of self-emulsifying systems with varying concentrations of Labrafil M 1944 CS (5\%-40\%, v/v), Cremophor RH $40(30 \%-70 \%, \mathrm{v} / \mathrm{v})$, Transcutol P $(0 \%-40 \%$, v/v), and morin $(1 \%-15 \%, w / w)$ was prepared. The samples were then vortexed and sonicated until oily liquid mixtures were obtained. Each sample was then added dropwise into distilled water $(\mathrm{v} / \mathrm{v}$, 1:100). The self-emulsifying performance of the generated samples was visually observed. Samples that showed drug precipitation or cracking were rejected. Samples that could easily spread in water and form a fine emulsion were judged as "good." Samples were labeled as "bad" when there was poor or no emulsion formation with immediate coalescence of oil droplets when stirring was stopped. ${ }^{22}$ Phase diagrams were plotted using the above criteria. Finally, the formulation composed of Labrafil M 1944 CS, Cremophor RH 40, and Transcutol P (3:5:3) with a drug loading of $10 \%(\mathrm{w} / \mathrm{w})$ was selected as the best one of its kind.

\section{Characterization of MPC-SNEDDS}

The droplet size and polydispersity index of the optimized morin-phospholipid complex-SNEDDS (MPC-SNEDDS) after dilution with distilled water $(1: 100, \mathrm{v} / \mathrm{v})$ were determined. Morphological features of the MPC-SNEDDS were observed by transmission electron microscope (TEM; Hitachi H-600; Hitachi Co, Tokyo, Japan). The sample was diluted with distilled water at a ratio of 1:100 and mixed by gentle shaking. One drop of the sample was then placed on copper grids, and any excess was drawn off with filter paper. 
The sample was negatively stained by $1 \%$ phosphomolybdic acid and then subjected to TEM observation.

\section{Pharmacokinetic studies in rats}

Animal experiments were conducted in accordance with the guidelines of the Institutional Animal Care and Use Committee of Sichuan University (Sichuan, China). Male Wistar rats (200-250 g) were provided by the Laboratory Animal Center of Sichuan University. Rats were randomly divided into four groups $(n=5)$ and fasted for 12 hours before administration of doses. One group was given intravenous morin saline solution $(1 \mathrm{mg} / \mathrm{kg})$. The other three groups were given morin suspension, MPC suspension, or the MPC-SNEDDS at $200 \mathrm{mg} / \mathrm{kg}$ by gavage. Morin suspension and MPC suspension were prepared by dispersing morin and MPC in 5\% sodium carboxymethyl cellulose solution, respectively.

Blood $(400 \mu \mathrm{L})$ was collected via tail vein into heparinized centrifuge tubes at prefixed time points after administration of these four dosage forms. Plasma was separated from whole blood by centrifugation $(1296 \times \mathrm{g}, 5$ minutes $)$ and stored at $4^{\circ} \mathrm{C}$ prior to analysis.

Rat plasma $(100 \mu \mathrm{L})$ was pipetted into a centrifuge tube, and then equal amounts of internal standard solution containing benzoic acid in methanol, methanol, and $25 \%$ hydrochloric acid were added sequentially. The resultant mixture was vortexed for 5 minutes, hydrolyzed in $50^{\circ} \mathrm{C}$ water bath for 0.5 hours, vortexed again for 1 minute, and then centrifuged $(12544 \times \mathrm{g}, 15$ minutes $)$. The supernatant $(100 \mu \mathrm{L})$ was injected directly into the HPLC column.

Using the HPLC condition mentioned above, the standard curve of morin concentration in plasma was established and showed good linearity $(r=0.9996)$ in the range of $0.48-48 \mu \mathrm{g} / \mathrm{mL}$. The standard curve equation of morin in serum was $\mathrm{Y}=0.2082 \mathrm{X}-0.0704$ ( $\mathrm{Y}$ stands for the concentration of morin, $\mathrm{X}$ stands for the peak area ratio of morin to internal standard). The recovery rates of morin from high, middle, and low concentration ranges were $97.8 \% \pm 0.012 \%, 95.1 \% \pm 0.033 \%$, and $91.2 \% \pm 0.047 \%$, respectively. The interday relative standard deviations were $2.18 \%, 2.60 \%$, and $2.27 \%$ respectively; and the intraday relative standard deviations were $3.40 \%, 3.53 \%$, and $5.10 \%$, respectively.

This method was then applied to the pharmacokinetic studies. The pharmacokinetic data were processed by DAS 2.0 software (Mathematical Pharmacology Professional Committee of China) to calculate the pharmacokinetic parameters. The absolute bioavailability $\left(\mathrm{F}_{\mathrm{a}}\right)$ and relative bioavailability $\left(\mathrm{F}_{\mathrm{r}}\right)$ were calculated according to the following equations:

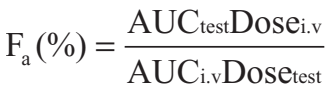

$$
\begin{aligned}
& \mathrm{F}_{\mathrm{r}}(\%)=\frac{\mathrm{AUC}_{\text {test }}}{\mathrm{AUC}_{\text {reference }}}
\end{aligned}
$$

AUC is the area under the plasma concentration-time curve from time 0 to the last sampling time for both equations.

\section{Statistical analysis}

Student's t test was used for statistical comparisons between the pharmacokinetic data of these three groups. A value of $P<0.05$ was considered statistically significant.

\section{Results and discussion Characterization of MPC}

The formation of MPC was confirmed by infrared spectra and X-ray diffraction. The infrared spectra of morin, phospholipid, MPC, and the physical mixture are shown
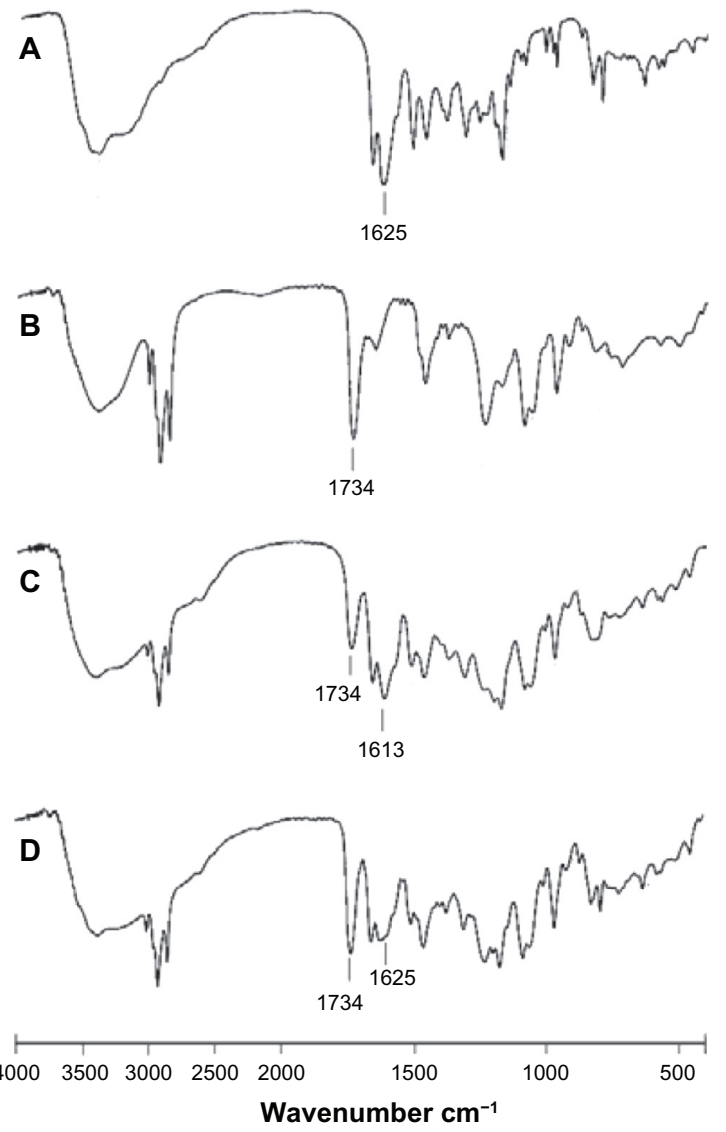

Figure I Infrared spectra of (A) morin; (B) phospholipids; (C) morin-phospholipid complex; and (D) physical mixture of morin and phospholipids. 
in Figure 1. There were significant differences between the spectrum of MPC (Figure 1C) and spectrum of the physical mixture (Figure 1D). The characteristic absorption peak of morin at $1625 \mathrm{~cm}^{-1}\left(\mathrm{v}_{\mathrm{C}-\mathrm{C}=\mathrm{O}}\right)$ was easily found in the spectrum of physical mixture. However, the absorption peak of morin had shifted up to $1613 \mathrm{~cm}^{-1}\left(\mathrm{v}_{\mathrm{C}=\mathrm{O}}\right)$ in the MPC spectrum (Figure 1C), indicating interactions between morin and phospholipids. On the other hand, the characteristic absorption peak of phospholipid was found to be at $1734 \mathrm{~cm}^{-1}$ $\left(\mathrm{v}_{\mathrm{OH}-\mathrm{C}=\mathrm{O}}\right)$ in spectrums of both the complex and the physical mixture. However, the absorption strength at $1734 \mathrm{~cm}^{-1}$ was lessened greatly by the formation of MPC, compared with that in the spectrum of phospholipid. In addition, there was no new peak observed in the MPC spectrum (Figure 1C).

Figure 2 shows the X-ray diffraction curves of morin, phospholipids, MPC, and the physical mixture. The morin powder diffraction pattern shown in Figure 2A has obvious sharp crystalline peaks. In contrast, phospholipids appeared to have an amorphous structure, as shown in

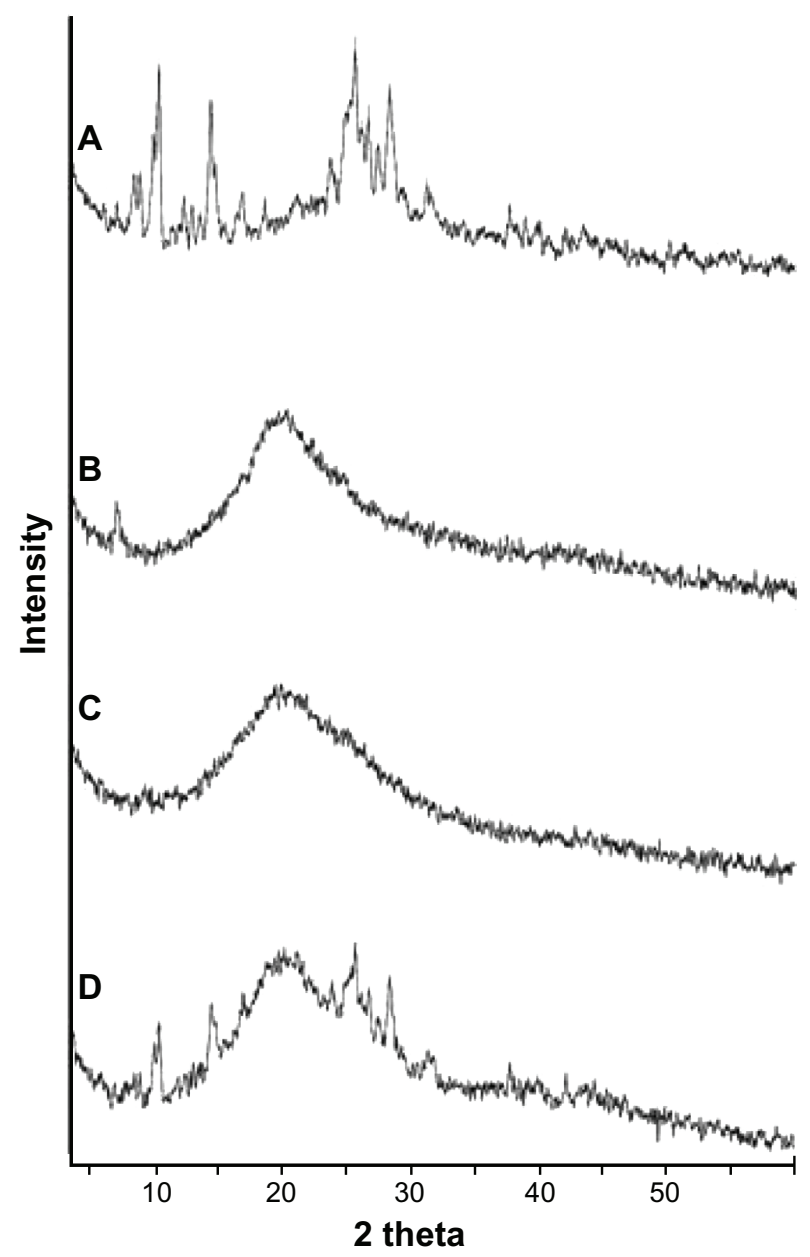

Figure 2 X-ray diffraction pattern of (A) morin; (B) phospholipids; (C) morinphospholipid complex; and (D) physical mixture of morin and phospholipids.
Table I Solubility of morin, MPC, and the physical mixture in water and n-octanol at room temperature

\begin{tabular}{lll}
\hline Sample & $\begin{array}{l}\text { Solubility in water } \\
(\mu \mathrm{g} / \mathrm{mL})^{\mathrm{a}}\end{array}$ & $\begin{array}{l}\text { Solubility in n-octanol } \\
(\mathbf{m g} / \mathbf{m L})^{\mathrm{a}}\end{array}$ \\
\hline Morin & $18.42 \pm 1.49$ & $11.96 \pm 6.71$ \\
MPC & $74.43 \pm 0.36$ & $>1000$ \\
Physical mixture & $34.40 \pm 0.01$ & $13.85 \pm 0.09$ \\
\hline
\end{tabular}

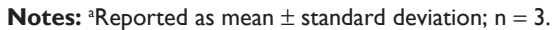

Abbreviations: MPC, morin-phospholipid complex; the mixture of phospholipids and morin, physical mixture.

Figure 2B. The crystalline structure of morin remained unchanged in the physical mixture (Figure 2D). However, as shown in Figure 2C, the crystalline peaks of MPC had disappeared, showing a structural characteristic similar to that of phospholipids. This was probably a consequence of noncovalent interactions between morin and phospholipids, such as hydrogen bonding and van der Waals forces. The morin molecule was "entrapped" in the polar head of phospholipids molecules, and thus its own crystalline characteristic was inhibited.

The content of morin in the complex determined by HPLC was $38.3 \%(\mathrm{w} / \mathrm{w})$. The result was concordant with the reactant ratio of morin and phospholipids.

\section{Solubility studies}

The enhancement on the liposolubility of morin by the formation of MPC was investigated. As shown in Table 1, the solubility of morin in the n-octanol increased significantly after the formation of MPC. However, the hydrophilicity and lipophilicity of morin was slightly improved by the phospholipids in physical mixture. The result indicated that the phospholipids exhibited beneficial effects on the solubility of morin after the formation of MPC. Simultaneously, the solubilities of morin and MPC in different types of oils were also investigated. Table 2 shows the enhancements on the solubility of morin in three types of oils after the formation of MPC. As shown in Table 2, the solubility of MPC was significantly higher than that of morin, suggesting that the formation of MPC

Table 2 Solubility of morin and MPC in different oils at room temperature

\begin{tabular}{|c|c|c|}
\hline \multirow[t]{2}{*}{ Type of oil } & \multicolumn{2}{|c|}{ Solubility $(\mathrm{mg} / \mathrm{mL})$} \\
\hline & $M P^{a}$ & Morin $^{a}$ \\
\hline Lauroglycol ${ }^{\mathrm{TM}} \mathrm{FCC}$ & $20.40 \pm 1.43$ & $3.15 \pm 0.056$ \\
\hline Labrafil ${ }^{\circledR}$ M I944 CS & $27.04 \pm 1.38$ & $5.83 \pm 0.12$ \\
\hline Lauroglycol 90 & $325.60 \pm 52.31$ & $83.30 \pm 2.34$ \\
\hline
\end{tabular}

Notes: aReported as mean \pm standard deviation; $\mathrm{n}=3$.

Abbreviation: MPC, morin-phospholipid complex. 
Table 3 Factors and levels of $L_{9}\left(3^{3}\right)$ orthogonal test

\begin{tabular}{llll}
\hline Run & Oil & Surfactant & Cosurfactant \\
\hline I & I (3) & I (3) & I (I) \\
2 & I (3) & $2(4)$ & $2(2)$ \\
3 & I (3) & $3(5)$ & $3(3)$ \\
4 & $2(4)$ & I (3) & $2(2)$ \\
5 & $2(4)$ & $2(4)$ & $3(3)$ \\
6 & $2(4)$ & $3(5)$ & I (I) \\
7 & $3(5)$ & I (3) & $3(3)$ \\
8 & $3(5)$ & $2(4)$ & I (I) \\
9 & $3(5)$ & $3(5)$ & $2(2)$ \\
\hline
\end{tabular}

Notes: Orthogonal experimental form of $L_{q}\left(3^{3}\right)$ was adopted to determine the ratios of oil, surfactant, and cosurfactant to be evaluated to a self-nanoemulsifying drug delivery system (SNEDDS) formulation.

could drastically facilitate the incorporation of morin into a SNEDDS. The solubility of morin in many other oils was less than $5 \mathrm{mg} / \mathrm{mL}$, even after MPC formation (data not shown).

\section{Development of MPC-SNEDDS}

As a simple, fast, and economical method, orthogonal design was used to screen the optimum blank SNEDDS. The ratios at which the oil, surfactants, and cosurfactants were mixed with each other are shown in Table 3. The droplet sizes of the resultant translucent nanoemulsions for each combination are listed in Table 4.
As shown in Table 4, all of the translucent nanoemulsions showed mean droplet sizes in the range of 20-100 $\mathrm{nm}$. Unfortunately, all samples were turbid when Lauroglycol 90 was used as oil phase. However, translucent nanoemulsions can be formed in the case of Labrafil M 1944 CS, which probably is due to its greater hydrophilicity and surfactant-like properties. ${ }^{23}$ In recent years, many drugs have been reported to exhibit significantly higher bioavailability after administration of an a long-chain triglyceride (LCT) solution formulation than a similar medium-chain triglyceride (MCT) solution, probably because of the more effective lymphatic drug transport or more efficient solubilization in the gastrointestinal tract by the long-chain lipid digestion product. ${ }^{24}$ With LCTs, there may be a potential advantage with using Labrafil M 1944 $\mathrm{CS}$ as the oil phase. Transcutol P and 1,2-propylene glycol were investigated as cosurfactants for their good solubility of morin (more than $1 \mathrm{~g} / \mathrm{mL}$ ). The results show that Transcutol P exhibited better self-nanoemulsifying ability due to its greater fluidity and compatibility than 1,2-propylene glycol. In addition, the formulation composed of Labrafil M 1944 CS, Cremophor RH 40, and Transcutol $P$ at a ratio of 3:5:3 was found to have the smallest particle size $(28.34 \pm 0.03 \mathrm{~nm})$, with a narrow size distribution $(0.067 \pm 0.006)$, after dilution. Thus, the combination of Labrafil M 1944 CS, Cremophor RH 40, and Transcutol $\mathrm{P}$ was chosen for further study.

Table 4 Droplet size and polydispersity index of SNEDDS formulations after dilution with distilled water at a ratio of I:I00

\begin{tabular}{|c|c|c|c|c|}
\hline System & Ratio & Particle size $(\mathrm{nm})^{\mathrm{a}}$ & Polydispersity index ${ }^{a}$ & Time of emulsification \\
\hline \multirow[t]{5}{*}{ Labrafil $^{\otimes}$ M I 944 CS/Cremophor ${ }^{\circledast}$ EL/Transcutol ${ }^{\circledR}$ P } & 3:3:1 & $35.21 \pm 0.79$ & $0.111 \pm 0.016$ & $1 \mathrm{~min} \sim 3 \mathrm{~min}$ \\
\hline & $3: 4: 2$ & $28.73 \pm 0.61$ & $0.098 \pm 0.013$ & $<1 \min$ \\
\hline & $3: 5: 3$ & $28.34 \pm 0.56$ & $0.067 \pm 0.006$ & $<I \min$ \\
\hline & $4: 3: 2$ & $33.85 \pm 1.21$ & $0.082 \pm 0.006$ & $<I \min$ \\
\hline & $4: 4: 3$ & $36.92 \pm 1.94$ & $0.222 \pm 0.031$ & $I \min \sim 3 \min$ \\
\hline \multirow[t]{8}{*}{ Labrafil M I 944 CS/Cremophor RH 40/Transcutol P } & $3: 3: 1$ & $39.93 \pm 0.87$ & $0.099 \pm 0.013$ & $<\mathrm{I} \min$ \\
\hline & $3: 4: 2$ & $30.48 \pm 0.74$ & $0.141 \pm 0.019$ & $<I \min$ \\
\hline & $3: 5: 3$ & $28.34 \pm 0.03$ & $0.079 \pm 0.003$ & $<I \min$ \\
\hline & $4: 3: 2$ & $39.45 \pm 1.17$ & $0.093 \pm 0.009$ & $<I \min$ \\
\hline & $4: 5: 1$ & $29.93 \pm 0.92$ & $0.114 \pm 0.017$ & $I \min \sim 3 \min$ \\
\hline & $4: 4: 3$ & $37.20 \pm 0.63$ & $0.228 \pm 0.039$ & $<1 \min$ \\
\hline & $5: 4: 1$ & $43.59 \pm 1.85$ & $0.101 \pm 0.008$ & $1 \min \sim 3 \min$ \\
\hline & $5: 3: 3$ & $76.75 \pm 2.16$ & $0.243 \pm 0.011$ & $1 \min \sim 3 \min$ \\
\hline \multirow[t]{4}{*}{ Lauroglycol ${ }^{\mathrm{TM}} \mathrm{FCC} /$ Cremophor RH 40/Transcutol P } & 3:3:1 & $35.69 \pm 1.22$ & $0.288 \pm 0.039$ & $<\mathrm{I} \min$ \\
\hline & $3: 4: 2$ & $34.38 \pm 0.97$ & $0.101 \pm 0.020$ & $<I \min$ \\
\hline & $3: 5: 3$ & $32.45 \pm 1.13$ & $0.253 \pm 0.04 I$ & $<I \min$ \\
\hline & $4: 4: 3$ & $41.93 \pm 1.52$ & $0.088 \pm 0.002$ & $<\mathrm{I} \min$ \\
\hline Lauroglycol FCC/Tween ${ }^{\circledast} 80 /$ I, 2-propylene glycol & $4: 4: 3$ & $37.93 \pm 2.90$ & $0.228 \pm 0.027$ & $<I \min$ \\
\hline Lauroglycol FCC/Cremophor EL/Transcutol P & 3:3:1 & $57.17 \pm 2.03$ & $0.388 \pm 0.046$ & $>3 \min$ \\
\hline Labrafil M 1944 CS/Tween 80/Transcutol P & $3: 5: 3$ & $97.40 \pm 4.58$ & $0.267 \pm 0.031$ & $<I \min$ \\
\hline
\end{tabular}

Notes: aReported as mean \pm standard deviation; $n=3$. 


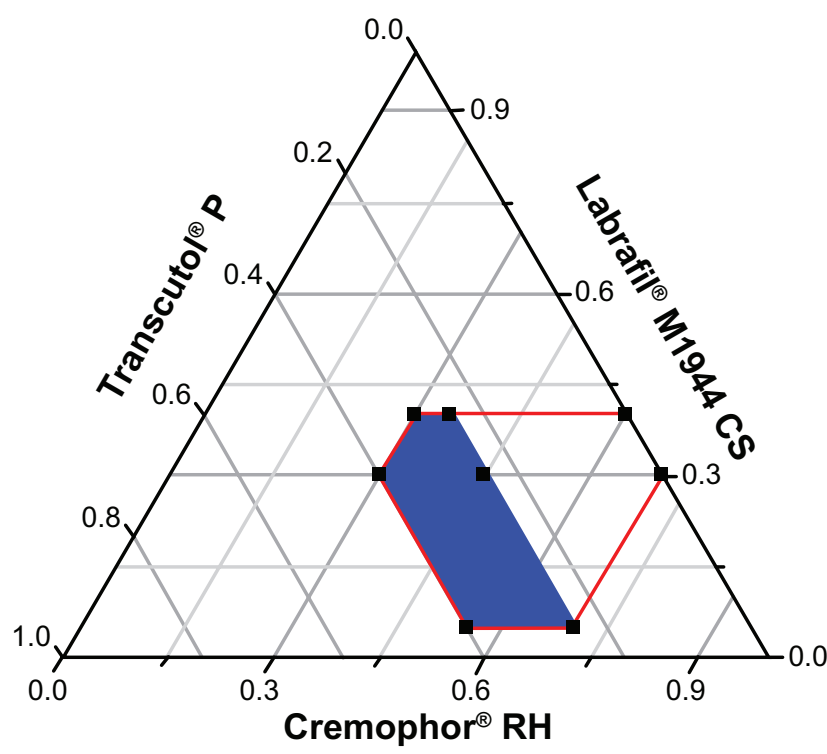

Figure 3 Ternary phase diagram of the SNEDDS formulation composed of the following: oil - Labrafil M 1944 CS; surfactant - Cremophor RH 40; and cosurfactant - Transcutol $P$, with a drug loading of $10 \%$.

Notes: The red outline represents the area explored for locating nanoemulsion region. The blue area represents nanoemulsion region.

The self-emulsifying performance of the selected SNEDDS with varying drug loading was accessed using ternary phase diagrams. The results show that the self-emulsification region of the formulation decreased on increased drug loading. To obtain a good balance between drug loading and efficient emulsification, the formulation composed of Labrafil M 1944 CS, Cremophor RH 40, and Transcutol P (3:5:3) with a drug loading of $10 \%(\mathrm{w} / \mathrm{w})$ was selected. The ternary phase diagram of the optimized formulation is shown in Figure 3.

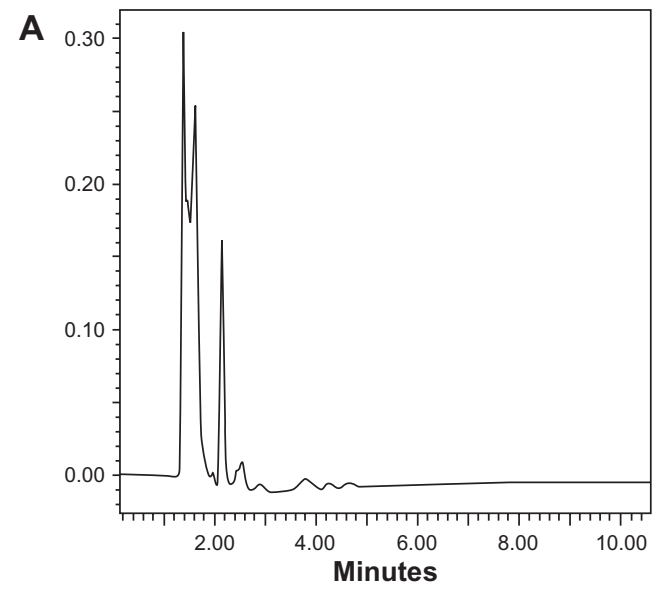

Figure 5 HPLC chromatographs of morin.

Notes: (A) Blank plasma; (B) plasma containing morin; internal standard: benzoic acid. Abbreviation: HPLC, high performance liquid chromatography.

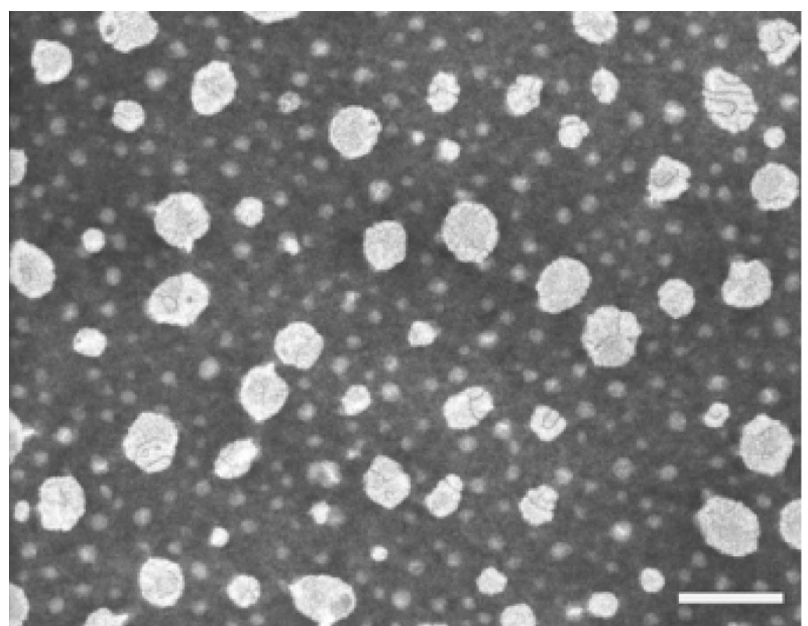

Figure 4 Transmission electron microscope image of MPC-loaded SNEDDS formulation.

Note: Scale bar $=200 \mathrm{~nm}$.

Abbreviations: MPC, morin-phospholipid complex; MPC-SNEDDS, morinphospholipid complex self-nanoemulsifying drug delivery system.

\section{Characterization of MPC-SNEDDS}

The prepared MPC-SNEDDS gave a mean particle size of $140.40 \mathrm{~nm}$ after dilution with distilled water. No precipitation of the drug was found when the sample was kept for 12 hours at $25^{\circ} \mathrm{C}$. As shown in the TEM image (Figure 4), the SNEDDS prepared in the optimized formulation appears to have spherical particles after dilution.

\section{Pharmacokinetic results}

The selectivity of the HPLC method was evaluated before conduction of the pharmacokinetic study. Figure 5 illustrates the chromatography of morin. Under the HPLC

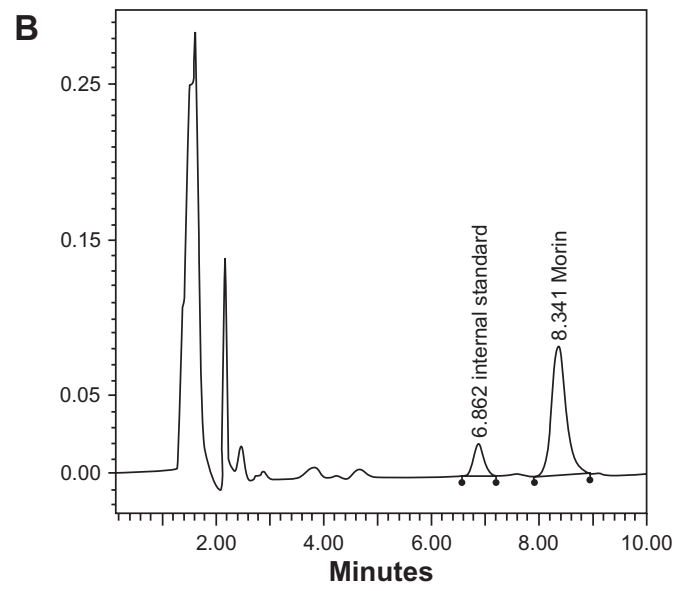




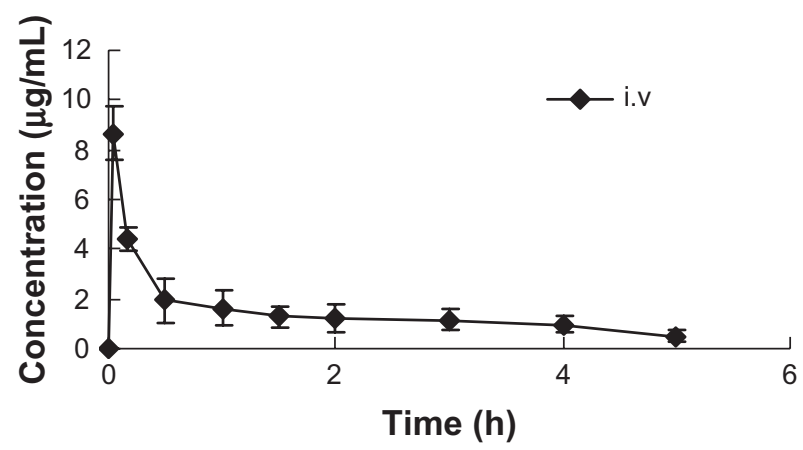

Figure 6 Plasma concentration-time curve after intravenous administration of morin in Wistar rats $(n=5)$ at a dose of $1 \mathrm{mg} / \mathrm{kg}$.

condition, the chromatograms show that there was no endogenous peaks interfering with morin or the internal standard. In addition, morin peaks can be separated well from benzoic acid peaks without any interference. The plasma concentration of morin after intravenous administration is shown in Figure 6. Intravenous data were used to calculate the absolute bioavailability of morin after oral administration of morin suspension, MPC suspension, and the MPC-SNEDDS. The plasma concentration-time curves of morin after oral administration are shown in Figure 7. As shown, the MPC-SNEDDS formulation-treated rat group had the highest concentration of morin in the plasma among the three groups during 0-5 hours (Figure 7). The oral pharmacokinetic parameters are summarized in Table 5. It was notable that the $\mathrm{C}_{\max }$ of the MPC-SNEDDS (28.60 $\mu \mathrm{g} / \mathrm{mL})$ group was significantly higher than the morin suspension $(5.53 \mu \mathrm{g} / \mathrm{mL})$ and MPC suspension (23.74 $\mu \mathrm{g} / \mathrm{mL})$ groups. Compared with the morin suspension, $\mathrm{T}_{\max }$ was prolonged from 0.48 to 0.77 hours and 1 hour for the MPC and the MPC-SNEDDS groups, respectively. Compared to the morin suspension, the mean relative bioavailabilities of the MPC and the MPC-SNEDDS were $541.73 \%$ and $722.84 \%$, respectively.

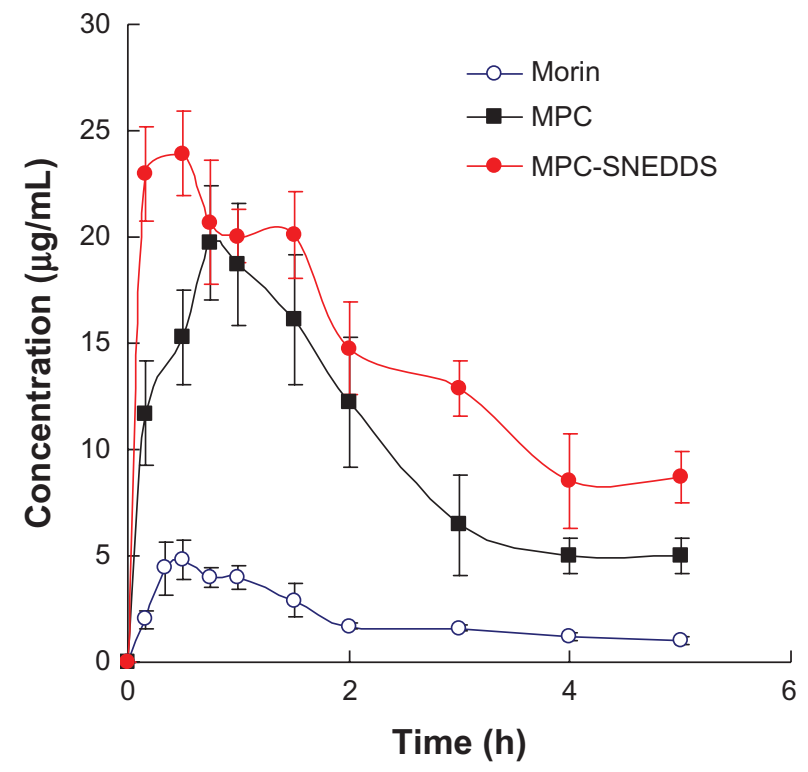

Figure 7 Mean plasma concentration-time profiles of morin in Wistar rats $(n=5)$ after oral administration of morin, MPC, and MPC-SNEDDS formulation at a dose of $200 \mathrm{mg} / \mathrm{kg}$.

Abbreviations: MPC, morin-phospholipid complex;MPC-SNEDDS, morin-phospholipid complex self-nanoemulsifying drug delivery system.

To the authors' knowledge, the in vivo tests are the first to report the absolute bioavailability of morin, which was only $0.45 \%$. It was assumed that the low oral bioavailability of morin may be caused by its low aqueous solubility $\left(0.25 \mathrm{mg} / \mathrm{mL}\right.$, distilled water, $\left.25^{\circ} \mathrm{C}\right), \mathrm{P}$-gp-mediated efflux, and low intestinal permeability $\left(\mathrm{P}_{\text {app }}=0.62 \mathrm{~nm} / \mathrm{s}\right){ }^{25}$ The absolute bioavailability of morin in rats was significantly increased by the MPC-SNEDDS compared to the morin and MPC suspensions. Reasons for this may include the following: (1) After oral administration, the intestinal mixing of the excipients, their digestion products, and bile components leads to the formation of mixed micelles and vesicles, thus improving the solubilization power of the gastrointestinal tract components, ${ }^{26}$ (2) The presence of a

Table 5 Bioavailability and pharmacokinetic parameters (mean \pm standard deviation) of morin in Wistar rats after oral administration of morin, MPC, and MPC-SNEDDS at a dose of $200 \mathrm{mg} / \mathrm{kg}(\mathrm{n}=5)$

\begin{tabular}{lllll}
\hline & Intravenous morin (I mg/kg) & Oral morin & Oral MPC & Oral MPC-SNEDDS \\
\hline $\mathrm{AUC}_{\text {0-t }}(\mathrm{mg} / \mathrm{L} \mathrm{h})$ & $11.89 \pm 0.21$ & $10.64 \pm 1.9$ & $57.64 \pm 17.19^{* * *}$ & $76.91 \pm 23.77^{* *, \ldots \#}$ \\
$\mathrm{MRT}_{\text {o-t }}(\mathrm{h})$ & $1.33 \pm 0.32$ & $1.91 \pm 0.28$ & $1.87 \pm 0.18$ & $2.12 \pm 0.0 \mathrm{I}^{\#}$ \\
$\mathrm{~T}_{\text {max }}(\mathrm{h})$ & $0.05 \pm 0.00$ & $0.48 \pm 0.17$ & $0.77 \pm 0.27^{*}$ & $1.00 \pm 0.47^{* *, \#}$ \\
$\mathrm{C}_{\text {max }}(\mu \mathrm{g} / \mathrm{mL})$ & $13.86 \pm 3.91$ & $5.53 \pm 2.07$ & $23.74 \pm 3.87^{* *}$ & $28.60 \pm 9.25^{* *, \#}$ \\
$\mathrm{~F}_{\mathrm{a}}(\%)$ & 100.00 & 0.45 & 2.42 & 3.24 \\
$\mathrm{~F}_{\mathrm{r}}(\%)$ & - & 100 & 541.73 & 722.84 \\
\hline
\end{tabular}

Notes: $* P<0.05$ compared with morin group; **P < 0.01 compared with morin group; ${ }^{*}>0.05$ MPC-SNEDDS group compared with MPC group; ${ }^{*} P<0.0$ I MPCSNEDDS compared with MPC group.

Abbreviations: $\mathrm{F}_{\mathrm{a}}$, absolute bioavailability; MRT, mean retention time; MPC, morin-phospholipid complex; MPC-SNEDDS, morin-phospholipid complex self-nanoemulsifying drug delivery system; $F_{r}$, relative bioavailability. 
SNEDDS can act as an intestinal permeability enhancer, which may result in increased absorption of morin via paracellular or transcellular routes, ${ }^{27,28}$ (3) Cremophor RH 40 and Transcutol $\mathrm{P}$ can inhibit the activity of the intestinal efflux pump (P-gp), and thus increase the absorption of morin in the gastrointestinal tract. Details on the potential effects of nonionic surfactants and cosurfactants on P-gp activity have also been published in other literature..$^{29,30}$

The interaction between the MPC-SNEDDS formulation and the biological environment is rather complicated, and consequently, the definite absorption mechanisms and the potential toxicity of the MPC-SNEDDS requires further investigation.

\section{Conclusion}

In this study, a MPC-loaded SNEDDS for oral administration was successfully developed. The phospholipid complex significantly improved the liposolubility of morin. The optimum SNEDDS formulation was a mixture of Labrafil M 1944 CS, Cremophor RH 40, and Transcutol P at a ratio of 3:5:3 (w/w/w). The absolute bioavailability of morin increased about 6.2-fold after oral administration of the MPCSNEDDS, compared with that of the morin suspension. This study represents initial efforts to develop a promising oral formulation of morin. In addition, it supports the technique of using a SNEDDS combined with the phospholipid complex technique as a promising delivery system to enhance the oral absorption of poorly water-soluble drugs.

\section{Acknowledgments}

This work was funded by the National Basic Research Program of China (973 program, No: 2009CB930300) and supported by the National Science and Technology Major Project of China (Grant No: 2009ZX09310-002). The authors would like to thank Rasa Hamilton (H Lee Moffitt Cancer Center, FL) for editorial assistance.

\section{Disclosure}

The authors report no conflicts of interest in this work.

\section{References}

1. Hsiu SL, Tsao CW, Tasi YC, et al. Determinations of morin, quercetin and their conjugate metabolites in serum. Biol Pharm Bull. 2001;24(8): 967-969.

2. Fang SH, Hou YC, Chang WC, et al. Morin sulfates/glucuronides exert anti-inflammatory activity on activated macrophages and decreased the incidence of septic shock. Life Sci. 2003;74(6):743-756.

3. Sivaramakrishnan V, Shilpa PN, Praveen Kumar VR, et al. Attenuation of N-nitrosodiethylamine-induced hepatocellular carcinogenesis by a novel flavonol-Morin. Chem Biol Interact. 2008;171(1):79-88.
4. Zhang R, Kang KA, Piao MJ, et al. Cellular protection of morin against the oxidative stress induced by hydrogen peroxide. Chem Biol Interact. 2009;177(1):21-27.

5. Kang DG, Moon MK, Sohn EJ, et al. Effects of morin on blood pressure and metabolic changes in fructose-induced hypertensive rats. Biol Pharm Bull. 2004;27(11):1779-1783.

6. Parihar VK, Prabhakar KR, Veerapur VP, et al. Anticlastogenic activity of morin against whole body gamma irradiation in Swiss albino mice. Eur J Pharmacol. 2007;557(1):58-65.

7. $\mathrm{Yu} \mathrm{Z}$, Fong WP, Cheng $\mathrm{CH}$. The dual actions of morin $\left(3,5,7,2^{\prime}, 4^{\prime}-\right.$ pentahydroxyflavone) as a hypouricemic agent: uricosuric effect and xanthine oxidase inhibitory activity. J Pharmacol Exp Ther. 2006; 316(1):169-175.

8. Cho YM, Onodera H, Ueda M, et al. A 13-week subchronic toxicity study of dietary administered morin in F344 rats. Food Chem Toxicol. 2006;44(6):891-897.

9. Hou YC, Chao PD, Ho HJ, et al. Profound difference in pharmacokinetics between morin and its isomer quercetin in rats. J Pharm Pharmacol. 2003;55(2):199-203.

10. Ko KT, Needham TE, Zia H. Emulsion formulations of testosterone for nasal administration. J Microencapsul. 1998;15(2):197-205.

11. Krishnaiah YSR. Pharmaceutical technologies for enhancing oral bioavailability of poorly soluble drugs. J Bioequiv Availab. 2010;2(2): 28-36.

12. Kang BK, Lee JS, Chon SK, et al. Development of self-microemulsifying drug delivery systems (SMEDDS) for oral bioavailability enhancement of simvastatin in beagle dogs. Int J Pharm. 2004;274(1-2):65-73.

13. ElnaggarYS, El-Massik MA, Abdallah OY. Self-nanoemulsifying drug delivery systems of tamoxifen citrate: design and optimization. Int $J$ Pharm. 2009;380(1-2):133-141.

14. Balakrishnan $\mathrm{P}$, Lee BJ, Oh DH, et al. Enhanced oral bioavailability of dexibuprofen by a novel solid self-emulsifying drug delivery system (SEDDS). Eur J Pharm Biopharm. 2009;72(3):539-545.

15. Zhang P, Liu Y, Feng NP, et al. Preparation and evaluation of selfmicroemulsifying drug delivery system of oridonin. Int J Pharm. 2008; 355(1-2):269-276.

16. Setthacheewakul S, Mahattanadul S, Phadoongsombut N, et al. Development and evaluation of self-microemulsifying liquid and pellet formulations of curcumin, and absorption studies in rats. Eur J Pharm Biopharm. 2010;76(3):475-485.

17. Date AA, Desai N, Dixit R, et al. Self-nanoemulsifying drug delivery systems: formulation insights, applications and advances. Nanomedicine (Lond). 2010;5(10):1595-1616.

18. Gursoy RN, Benita S. Self-emulsifying drug delivery systems (SEDDS) for improved oral delivery of lipophilic drugs. Biomed Pharmacother. 2004;58(3):173-182.

19. Maiti K, Mukherjee K, Gantait A, et al. Curcumin-phospholipid complex: preparation, therapeutic evaluation and pharmacokinetic study in rats. Int J Pharm. 2007;330(1-2):155-163.

20. Cui F, Shi K, Zhang L, et al. Biodegradable nanoparticles loaded with insulin-phospholipid complex for oral delivery: preparation, in vitro characterization and in vivo evaluation. J Control Release. 2006;114(2): $242-250$.

21. Yanyu X, Yunmei S, Zhipeng C, et al. The preparation of silybinphospholipid complex and the study on its pharmacokinetics in rats. Int J Pharm. 2006;307(1):77-82.

22. Craig DQM, Barker SA, Banning D, et al. An investigation into the mechanisms of self-emulsification using particle size analysis and low frequency dielectric spectroscopy. Int J Pharm. 1995;114(1): $103-110$.

23. DateAA, Nagarsenker MS. Design and evaluation of self-nanoemulsifying drug delivery systems (SNEDDS) for cefpodoxime proxetil. Int $J$ Pharm. 2007;329(1-2):166-172.

24. Porter CJH, Pouton CW, Cuine JF, Charman WN. Enhancing intestinal drug solubilisation using lipid-based delivery systems. Adv Drug Deliv Rev. 2008;60(6):673-691. 
25. Tian XJ, Yang XW, Yang X, et al. Studies of intestinal permeability of 36 flavonoids using Caco-2 cell monolayer model. Int J Pharm. 2009; 367(1-2):58-64.

26. MacGregor KJ, Embleton JK, Lacy JE, et al. Influence of lipolysis on drug absorption from the gastro-intestinal tract. Adv Drug Deliv Rev. 1997;25(1):33-46.

27. Aungst BJ. Intestinal permeation enhancers. J Pharm Sci. 2000;89(4): 429-442.

28. Hauss DJ. Oral lipid-based formulations. Adv Drug Deliv Rev. 2007; 59(7):667-676
29. Buggins TR, Dickinson PA, Taylor G. The effects of pharmaceutical excipients on drug disposition. Adv Drug Deliv Rev. 2007;59(15): 1482-1503.

30. Constantinides PP, Wasan KM. Lipid formulation strategies for enhancing intestinal transport and absorption of P-glycoprotein (P-gp) substrate drugs: in vitro/in vivo case studies. J Pharm Sci. 2007;96(2): $235-248$.

\section{Publish your work in this journal}

The International Journal of Nanomedicine is an international, peerreviewed journal focusing on the application of nanotechnology in diagnostics, therapeutics, and drug delivery systems throughout the biomedical field. This journal is indexed on PubMed Central, MedLine, CAS, SciSearch $\AA$, Current Contents ${ }^{\circledR} /$ Clinical Medicine,
Journal Citation Reports/Science Edition, EMBase, Scopus and the Elsevier Bibliographic databases. The manuscript management system is completely online and includes a very quick and fair peer-review system, which is all easy to use. Visit http://www.dovepress.com/ testimonials.php to read real quotes from published authors. 